\title{
La inversión en capital humano de los jóvenes gallegos: ¿sigue siendo rentable la educación?
}

\author{
M $^{a}$ Jesús Freire Seoane ${ }^{a}$ \\ Universidade da Coruña
}

\section{Mercedes Teijeiro Álvarez \\ Universidade da Coruña}

\section{RESUMEN}

Una parte importante de la salud de una economía se refleja en la evolución de su mercado de trabajo. Conocer las variables que más afectan es fundamental para realizar un diagnóstico de la inserción laboral. Pese al amplio uso en las investigaciones sobre capital humano de los rendimientos de la educación, existen pocos estudios en donde se profundice en este tema centrándose en la problemática particular de los jóvenes. Esta investigación tiene como objetivo analizar los rendimientos privados que los jóvenes obtienen de las inversiones en educación tomando como referencia las ecuaciones de ingresos de Mincer.

${ }^{a} M^{a}$ Jesús Freire Seoane. Directora do Observatorio Ocupacional. Centro Universitario de Riazor (CUR). Almirante Lángara s/n, 15011 A Coruña. Tel: +34 981167000 ext: 2651 Fax: 981167106. Correo electrónico: maje@udc.es

${ }^{\mathrm{b}}$ Mercedes Teijeiro Álvarez. Profesora del Departamento de Análisis Económico y Administración de Empresas. Facultad de Ciencias Económicas y Empresariales. Campus de Elviña s $\mathbf{n}$, 15071 A Coruña. Tel: +34 981167000 ext: 2470. Correo electrónico: mercedes.teijeiro@ udc.es

Recibido: marzo 2010/ Aceptado: junio 2010 
El estudio se lleva a cabo con microdatos procedentes de una encuesta realizada en el año 2008 en diferentes zonas geográficas de Galicia (Bergantiños, Costa da Morte y Ferrolterra). La población objeto de estudio son jóvenes trabajadores con edades comprendidas entre 16 y 34 años, ambos incluidos. En el análisis se profundiza en una serie de rasgos básicos de los individuos de la muestra, los más significativos son: el sexo, el nivel de estudios, la edad, la experiencia, el trabajar por cuenta ajena y la preferencia de ubicación del trabajo cercano al domicilio.

Palabras clave: inversión en capital humano, ecuaciones de ingresos, jóvenes trabajadores, nivel de estudios.

Clasificación JEL: I20, I22, J24.

\begin{abstract}
An important part of the health of any economy is reflected in the evolution of its labour market. To determine the characteristics of the main variables is essential for making a diagnosis of labour insertion. Despite returns to education wide use in the investigation, very few studies exist which expand on this topic, focusing on the particular problems of youth population. This research aims to analyze the private performance that young people get from investment in education, taking by reference the Mincer earnings equations.

The study is conducted with micro data from a survey performed in 2008 in different geographical areas (Bergantiños, Costa da Morte and Ferrolterra) of the Autonomous Community of Galicia. The target population are young workers aged between 16 and 34 years, last both inclusive. The analysis explores a number of basic features of the individuals in the sample, the most significant are: gender, educational level, age, experience, paid employment and the preference of work locations nearby at home.

Keywords: human capital investment, earning equations, youth, Galician municipality
\end{abstract}

JEL Classification: I20, I22, J24 


\section{INTRODUCCIÓN}

La rentabilidad de la educación es uno de los temas más debatidos en economía de la educación. La mayor parte de las teorías existentes coinciden en considerar la formación como un bien de inversión que aumenta la probabilidad de que un individuo perciba salarios más elevados. La decisión de adquirir educación adicional por parte del joven va a estar influida por los costes individuales directos y de oportunidad de esta opción, y por las repercusiones que puede tener en las oportunidades de empleo y en los niveles futuros de renta.

Pero la literatura, tanto teórica como empírica, apunta a que otras características como el sexo, la edad, la experiencia, la habilidad innata, así como la propia actitud del joven hacia el trabajo (su predisposición a aprender, a innovar contenidos, etc) pueden ser cruciales para que una persona consiga un puesto de trabajo que sea estable y que esté bien remunerado.

Este artículo describe y aplica la metodología empleada en la literatura sobre rendimientos de la educación al colectivo de los jóvenes entre 16 y 34 años, tomando como referencia la «ecuación de ingresos de Mincer» y teniendo en cuenta los posibles sesgos que aparecen en la misma, así como el efecto que produce en los rendimientos de la educación la desagregación de la escolaridad por nivel de estudios completado.

El trabajo se estructura en 6 apartados. En el segundo, se realiza una revisión de la literatura existente, haciendo especial énfasis en el marco teórico de las ecuaciones de ingresos. En el tercero se revisan los resultados de algunos de los principales estudios empíricos sobre rendimientos de la educación existentes en nuestro país. En el cuarto se describen las especificaciones del modelo y las principales características de la base de datos utilizada. En el quinto se muestran los resultados de la estimación de los efectos de la educación y la experiencia laboral en los salarios de los jóvenes. Finalmente, el trabajo se cierra con una recopilación de las principales conclusiones.

\section{Marco teórico de las ECUACIONES DE INGRESOS}

El estudio de las relaciones existentes entre el nivel de educación formal y las ganancias de un individuo ha sido un tema constante en la literatura económica, incluso remontándose hasta mediados del siglo XVIII aparece la obra de Cantillon (1755) en donde se justificaba el pago de los salarios más altos para los trabajadores más cualificados y, posteriormente, la obra de Smith (1776) que retoma las ideas anteriores y que 
se puede considerar como el precursor de la moderna teoría del capital humano. Sin embargo, es necesario esperar hasta principios de los años 60 del pasado siglo con los trabajos de Schultz (1961), Becker(1964) y Mincer (1974), para que se pueda hablar de un nuevo paradigma sobre la educación.

El capital humano se adquiere principalmente a través de la educación formal en la escuela, en el instituto, en la universidad; pero también, como educación informal, en el mercado de trabajo a través de la experiencia y la formación. En definitiva, el término capital humano es una expresión que hace referencia a la capacidad productiva del individuo, de forma similar a la de cualquier otro stock de capital en cuya producción y mantenimiento se incurre en costes pero del que se esperan también obtener ingresos (Salas, 2008). Según la teoría del capital humano, la educación aumenta la productividad de las personas y, por tanto, mejora las oportunidades de obtener mayores rentas salariales futuras (Becker, 1964). Siguiendo este enfoque, uno de los principales componentes del capital humano es que se acumula durante los años de educación formal y que existe una relación clara entre ingresos y educación. Cada individuo en función de su capacidad o habilidad, debería invertir en educación hasta el punto en que la tasa interna de rentabilidad se igualase al tipo de interés de mercado.

En contraste a esta hipótesis de la educación como un proceso de inversión en capital humano, existe la corriente credencialista cuya base teórica plantea la educación como un proceso de señalización para los demandantes de trabajo más que como una inversión en capital humano. Esta visión tiene como resultado el que los años de educación adquiridos que no vienen seguidos de la adquisición de un título no tengan ningún efecto en el ingreso del individuo. Estas interpretaciones no son excluyentes en la realidad, ya que los rendimientos de la educación tienen estos dos componentes teóricos, el de inversión en capital humano y el de señalización.

La OCDE (1998) distingue, al menos, tres enfoques para medir el stock de capital humano:

a) El nivel de estudios de la población adulta, medido como el promedio de años de educación formal completada, o como el porcentaje de adultos que ha terminado cada ciclo educativo. Este primer enfoque consiste en utilizar el nivel más alto de educación completado por cada individuo como una aproximación a la cantidad de capital humano.

b) Evaluación de las habilidades de la población adulta, mediante la valoración de las aptitudes de los mismos. Los resultados del IALS (International Adult Literacy Survey) proporcionan un nuevo enfoque en la medición de las habilidades y competencias dentro de un contexto internacional. 
c) Medir el capital humano vía salarios. Este último enfoque estima el valor agregado del stock de capital humano observando las diferencias en los ingresos de la población adulta, asociadas con características individuales particulares. Con este enfoque se solventaría el problema existente en los anteriores apartados de no tener en cuenta el aprendizaje adquirido a través de la experiencia laboral u otras vías de formación al margen de la educación reglada.

A partir de los trabajos de Becker han aparecido múltiples aportaciones en materia de economía de la educación, en donde se estiman las tasas de rendimiento de las inversiones en formación. El interés del tema reside en la búsqueda de la eficiencia en la distribución de los recursos escasos, lo que permitirá, por un lado, detectar problemas de asignación de recursos en el sistema educativo y, por otro, facilitar información a nivel particular sobre la existencia de rendimientos monetarios positivos asociados a la adquisición de educación adicional. Esta información permitirá a los individuos tomar decisiones más racionales sobre la continuación o no de sus estudios una vez finalizada su educación obligatoria. No es de extrañar, por tanto, que la acumulación del capital humano, así como la inversión en educación hayan sido uno de los temas principales en la determinación de los ingresos individuales y sean un factor a tener en cuenta en el crecimiento económico de un país.

El interés por analizar y cuantificar los rendimientos de la educación ha dado origen a una extensa literatura en el ámbito internacional. La herramienta empírica utilizada en la mayoría de estos trabajos ha sido la ecuación minceriana de ingresos, a través de la cual se estima el impacto de un año adicional de estudios en las rentas laborales de los individuos (Psacharopoulos, 1994; Cohn y Addison, 1998; Asplund y Pereira, 1999, Harmon et al, 2001, 2003).

La ecuación tradicional de Mincer, estima por mínimos cuadrados ordinarios $(\mathrm{MCO})^{1}$ un modelo semilogarítmico, usando como variable dependiente el logartimo de los ingresos y como variables independientes los años de educación, la experiencia laboral y el cuadrado de ésta (ecuación 1). Los datos utilizados para su estimación provienen tradicionalmente de datos transversales.

$$
\operatorname{Ln}(\mathrm{Y})=\xi_{0}+\xi_{1} \mathrm{~S}+\xi_{2} \operatorname{Exp}+\xi_{3} \operatorname{Exp}^{2}+0
$$

Donde:

${ }^{1}$ En adelante Mínimos Cuadrados Ordinarios se reflejará como MCO. 
Y son los ingresos del individuo

S es el número de años de educación formal completada

Exp son los años de experiencia laboral

$\operatorname{Exp}^{2}$ son los años de experiencia laboral al cuadrado

$\diamond$ es el término de perturbación aleatoria que se distribuye según una Normal $\left(0, \leftarrow_{0}^{2}\right)$

La función de ingresos de Mincer postula el paralelismo de los perfiles del logaritmo del ingreso con respecto a los distintos niveles de educación (Sapelli, 2003), si suponemos cumplidas las hipótesis bajo las que se desarrolla la función de Mincer $^{2}$ el valor del coeficiente de los años de educación formal se interpreta como la tasa de rendimiento media de un año adicional de estudio poseído por los trabajadores. Por otro lado, y teniendo en cuenta la teoría de los perfiles de edad-ingresos (conforme aumenta la experiencia, los ingresos individuales aumentan, pero cada año de experiencia tiene un efecto sobre los ingresos menor que el anterior), se espera que al ser la función cóncava con relación a la experiencia, la estimación de $\}_{2}$ sea positiva y la de $\}_{3}$ sea negativa.

La popularidad de esta ecuación se ha hecho extensible en múltiples ámbitos debido a su facilidad de aplicación y a su gran capacidad para generar resultados razonables para distintos conjuntos de datos (diferentes países, distintos instantes temporales, etc). Es preciso comentar que la ecuación 1 se construye bajo los supuestos del modelo neoclásico del funcionamiento del mercado de trabajo: las empresas conocen la productividad marginal de cada trabajador y el proceso competitivo hace que los salarios dependan de dicho nivel de productividad.

Con el tiempo las aportaciones científicas demostraron que en la medición del rendimiento de la educación estimado por MCO (ecuación minceriana de ingresos) existían varios sesgos, Griliches (1977), identificó los siguientes:

a) Existencia de determinadas variables omitidas en la ecuación de Mincer como la habilidad del individuo. Si el término de perturbación incluye entre otros elementos la habilidad del individuo, y se cumple que las personas con mayor habilidad son las que deciden elegir el mayor nivel educativo, esto provocaría

${ }^{2}$ a) Los costes de la inversión en educación son, únicamente, los costes de oportunidad (los ingresos que dejan de percibirse).

b) El tiempo que un individuo permanece en el mercado laboral es independiente del nivel de estudios alcanzado, y se supone que su permanencia en el mismo es continua.

c) Los individuos comienzan a trabajar inmediatamente después de finalizar sus estudios. 
estimaciones inconsistentes, pues la perturbación aleatoria y el nivel educativo (parámetro S) estarían correlacionados y esto daría, como resultado, que se sobreestimase el rendimiento de la educación.

b) Medición incorrecta de la cantidad de educación. La ecuación de Mincer (1) implica que existe una única tasa de rendimiento de la educación, sin embargo, tanto la teoría como la evidencia empírica lo contradicen, sugiriendo representar la escolaridad en forma más desagregada y flexible, con objeto de recoger una de las formas más adecuadas de los retornos al proceso de inversión en educación (Sapelli, 2009). El no recoger correctamente el nivel educativo estaría provocando una infraestimación en el verdadero rendimiento.

c) Tratamiento de la educación como una variable exógena. Diversos estudios han demostrado que la variable educación puede no ser exógena estando afectada por diferentes variables como puede ser los costes directos de la educación, los costes de oportunidad, los ingresos futuros, las imperfecciones del mercado de capitales o incluso la importancia del motivo consumo. El no considerar la endogeneidad de la educación producirá que las estimaciones realizadas por la técnica de MCO proporcione estimadores sesgados e inconsistentes del rendimiento de la educación ${ }^{3}$. Si se demuestra que la variable educación es endógena, el método más apropiado para calcular sus rendimientos sería estimarlo por «mínimos cuadrados en dos etapas» (MC2E). En este método la variable que causa la autocorrelación se elimina sustituyéndola por una variable instrumental (ecuación 2).

$$
\left.\begin{array}{c}
\left.\left.\left.\left.\operatorname{Ln}\left(\mathrm{Y}_{\mathrm{i}}\right)=\right\}_{0}+\right\}_{1} \mathrm{~S}_{\mathrm{i}}+\right\}_{2} \operatorname{Exp}_{\mathrm{i}}+\right\}_{3} \operatorname{Exp}_{\mathrm{i}}^{2}+\vartheta_{\mathrm{i}} \\
\mathrm{S}_{\mathrm{i}}=\mid{ }^{\prime} \mathrm{V}_{\mathrm{i}}+\mathrm{v}_{\mathrm{i}}
\end{array}\right\}
$$

Donde:

$\mathrm{V}_{\mathrm{i}}$ es un vector que recoge aquellas variables que pueden afectar a los años de educación formal conseguida pero que no están correlacionados con el término de perturbación $\diamond$.

$\mathrm{v}_{\mathrm{i}}$ es una variable aleatoria con media cero y varianza constante.

3 Autores que han detectado la endogeneidad de la educación son, entre otros: Kodde y Ritzen, 1984; Parsons, 1974; Wallace y Ihnen, 1975; Kodde y Ritzen, 1985, Levhari y Weiss, 1974; Eaton y Rosen, 1980 . 
A mayores de los sesgos recogidos por Griliches, existen otros dos sesgos de importancia, el primero tiene que ver con el uso de información de corte transversal para construir cohortes artificiales, en vez de utilizar cohortes efectivas a lo largo del tiempo. Esta forma de trabajar que está obviamente determinada por la disponibilidad de información, asume un comportamiento de las condiciones económicas muy estable y un mecanismo de generación de expectativas, asociado al proceso de inversión en capital humano, estático.

Y el segundo, consiste en la selección muestral, el problema derivado de este sesgo surge de sólo poder observar las rentas salariales de aquellos individuos ocupados. Aparece principalmente, cuando ciertos individuos se encuentran sistemáticamente más presentes en un determinado grupo frente a otro (Heckman,1979). Para corregir el sesgo de selección se recomienda utilizar el procedimiento de dos etapas propuesto por Heckman que consiste en especificar la probabilidad que tiene el individuo de estar ocupado, a partir de un modelo probit (ecuación 3).

$$
\mathrm{P}\left(\mathrm{Z}_{\mathrm{i}}=1\right)=\mathrm{P}\left(\boldsymbol{*}^{\prime} \mathrm{W}_{\mathrm{i}}+\mathrm{u}_{\mathrm{i}}>0\right)=\psi\left(\frac{\mathrm{rW}_{\mathrm{i}}}{\overleftarrow{\mathrm{i}}}\right)
$$

Donde:

$\mathrm{Z}_{\mathrm{i}}$ una variable ficticia que tomará el valor 1 cuando el individuo esté ocupado y cero en caso contrario.

$\mathrm{W}_{\mathrm{i}}$ es un vector de características individuales que influyen en la probabilidad de estar ocupado.

$\mathrm{u}_{\mathrm{i}}$ es el término de error que se distribuye según una normal de media cero.

El coeficiente lambda refleja el efecto que tiene en los salarios dejar fuera de la muestra a aquellas personas que podrían trabajar pero que, por el hecho de no trabajar en el momento de la encuesta, no se dispone de información acerca de sus salarios. El hecho de que * sea significativo nos indica que la muestra no es aleatoria y que su inclusión en la ecuación de salarios nos permitirá obtener estimadores consistentes (ecuación 4).

$$
\text { Ln } \left.y_{i}=\right\}^{\prime} x_{i}+\leftarrow_{i}+z_{i}
$$

Donde:

$\leftrightarrow$ es el coeficiente de correlación entre las perturbaciones de las ecuaciones que definen la probabilidad de ocupación y la función de ganancias original.

$\mathrm{X}_{\mathrm{i}}$ es el vector de variables anteriores.

$\geq_{i}$ es un término de perturbación aleatoria que se distribuye según una Normal $\left(0, \leftarrow_{w}^{2}\right)$. 
Buena parte de la literatura reciente se ha centrado en estimar el rendimiento libre de los sesgos que pudiera captar cuando no se tienen en cuenta estos problemas. Destacan a este respecto, los trabajos de Blackburn y Neumark (1993,1995), Angrist y Krueguer (1994, 1995), Card (1994, 2001), Murnane et al. (1995), Ashenfelter y Rouse (1997), Heckman y Vytlacil (2000), Barceinas et al. (2000, 2001) y Sapelli (2003).

\section{LAS ECUACIONES DE INGRESOS. RESULTADOS A NIVEL NACIONAL}

En este apartado se realiza un resumen de los resultados de algunos de los principales estudios empíricos llevados a cabo a nivel nacional sobre el tema objeto de estudio.

Calvo (1988) utiliza datos de la Encuesta de Presupuestos Familiares de 1980/81. Los resultados obtenidos indican incrementos relativamente importantes de renta a medida que aumenta el nivel educativo y la experiencia en el puesto de trabajo. Especificando por niveles educativos, los resultados muestran que los universitarios ganan el doble que aquellos que no tienen ningún tipo de estudios, un $70 \%$ más que los que solamente completaron la enseñanza primaria y un $40 \%$ más que los que finalizaron los estudios de bachillerato. Barceinas et al. (2000) trabajan con la misma encuesta para el mismo período y obtienen una rentabilidad de la educación del 5,9\%.

Alba y San Segundo (1995) utilizan datos de la Encuesta Piloto de Ingresos de 1990. En esta investigación se estima mediante la ecuación minceriana los rendimientos de los individuos asalariados y autoempleados. Los resultados obtenidos muestran que el rendimiento en el caso de los hombres es del 7,7\% y en las mujeres del 9,7\%.

Vila y Mora (1996) usando la misma encuesta para el mismo período obtienen una rentabilidad del 5,3\% para cada año de educación formal. Oliver et al. (1998) con la misma base de datos hallan una tasa interna de rentabilidad privada (TIR) para los estudios primarios, secundarios y superiores del $14,2 \%, 5,9 \%$ y $14,2 \%$, respectivamente.

San Segundo (1996), utiliza los datos provenientes de la Encuesta de Presupuestos Familiares (EPF) de 1980-1981 y 1990-1991. En esta investigación, para minimizar los problemas de sesgos de selección en las muestras se analizan únicamente las rentas de hombres asalariados. Sus resultados evidencian que la rentabilidad de los estudios universitarios no parece disminuir en los años analizados, a pesar de que se haya duplicado el número de titulados superiores. Si bien es cierto, que las conclusiones obtenidas permiten señalar que el mercado de trabajo está haciendo más atractivas las titulaciones de primer ciclo. 
Lasibillle y Navarro (1998) utilizando la Encuesta de Presupuestos Familiares, estudian la evolución de los rendimientos de la educación en España comparando los rendimientos para los años 1980/81 y 1990/91. Sus conclusiones muestran que entre 1981 y 1991 se observa en general un descenso del beneficio marginal de la formación de los hombres asalariados, sobre todo de los universitarios de ciclo largo. La excepción a esta pauta la constituye el aumento de la rentabilidad de los estudios universitarios de ciclo corto. A la misma conclusión (incremento del rendimiento marginal en las diplomaturas y decremento en las licenciaturas) llegan Vila y Mora (1998) para el mismo período y colectivo de varones y asalariados.

Blanco y Pons (2000) a partir de la Encuesta de Estructura, Conciencia y Biografía de Clase de 1991, estiman las tasas de rendimiento de los asalariados (16-65 años) mediante la ecuación minceriana incluyendo la habilidad innata y realizando la corrección por autoselección. Los autores demuestran que las tasas de rendimiento cuando se tiene en cuenta la habilidad innata son menores que los resultados sin tener en cuenta dicha habilidad; aún así, los individuos con mayor nivel educativo siguen teniendo una retribución mayor que la obtenida por los individuos con menor nivel.

Barceinas et al.. (2000) a partir del Panel de Hogares de la Unión Europea de 1994, estiman por MCO los rendimientos de la educación para los trabajadores a tiempo completo con unos rendimientos del 7,5\% para los hombres y 8,3\% para las mujeres. Arrazola y de Hevia (2001) realizan con la misma base de datos un estudio para los asalariados a tiempo completo y utilizan el modelo minceriano tradicional con corrección por autoselección empleando el método bietápico de Heckman. Los resultados son algo inferiores en el caso de los hombres del 6,4\% y en las mujeres del 7,4\%.

Barceinas et al. (2001) a partir de la Encuesta de Estructura Salarial de 1995 y utilizando la ecuación de Mincer, obtienen una rentabilidad de la educación del 8,2\% para los hombres y del 8,3\% para las mujeres. También utiliza la misma base de datos para realizar un estudio comparativo entre los resultados obtenidos con la estimación por MCO y la estimación con variables instrumentales (la variable utilizada es un índice de la reforma educativa de 1970), sus resultados muestran un rendimiento bastante mayor en el caso de la estimación con variables instrumentales un $8,8 \%$, frente a un $6,2 \%$ con MCO.

Caparrós et al. (2001) con datos del Panel de Hogares de la Unión Europea compara los rendimientos de la educación para los años 1994 y 1996 utilizando el modelo ortodoxo de Mincer, aunque corrigiendo el posible sesgo de selección muestral mediante la estimación del modelo en dos etapas de Heckman. Para el caso de España, los resultados para el año 1994 son de unos rendimientos del 8,1\% y del 9,5\% para hombres y mujeres, respectivamente. Para el año 1996 son del 6,6\% y del 9,1\%, se detecta una reducción en el rendimiento en los dos grupos. 
Pons y Gonzalo (2002) estiman los rendimientos de la educación en España para varones asalariados utilizando el Panel de Hogares de la Unión Europea para 1994 y usando el método de variables instrumentales (las variables utilizadas son el background familiar y la disponibilidad de universidad), sus resultados muestran una estimación de los rendimientos del $10 \%$ frente a un resultado del 6,4\% en la estimación por MCO.

Arrazola et al. (2003), utilizando la misma base de datos para el mismo año estiman las tasas internas de rentabilidad de los estudios básicos $(10,3 \%)$, de los estudios de bachillerato $(7,7 \%)$ y de los estudios universitarios de ciclo largo $(9,7 \%)$. En el mismo trabajo estimando por MCO la tasa de rendimiento de la inversión en educación arroja un resultado del 6,5\% y aumenta hasta el 8,2\% si el método utilizado es el de variables instrumentales (las variables utilizadas son los efectos en los logros educativos alcanzados con la introducción de la Ley General de Educación de 1970).

Salas (2004) realiza una encuesta entre graduados en la Universidad de Granada para los años 1996-1997 con la condición de estar colegiado, ocupado y asalariado. Estima los rendimientos de la educación mediante el método de Mincer, con corrección por autoselección y con tratamiento de endogeneidad, los resultados son de $8,6 \%$ sin selección muestral y sin contemplar la endogeneidad de la educación y pasan a ser del 13,6\% cuando considera la endogeneidad de la educación. La selección muestral no resultó significativa.

Marcenaro y Navarro (2005) usando los datos del Panel de Hogares de la Unión Europea para 1994 calculan las tasas de rendimiento privado de la educación por sexo haciendo uso del modelo ortodoxo de experiencia de Mincer, en el que se introduce un término de corrección de sesgo de selección muestral, según el procedimiento de dos etapas de Heckman. Los resultados más significativos son el incremento paulatino del rendimiento de la educación en España, a medida que se avanza en los niveles educativos y el mayor rendimiento que tiene la educación para las mujeres.

La conclusión más clara de la revisión de los trabajos recientes realizados en España es que cada vez son más depurados y fiables y que atribuyen, de forma general, rendimientos positivos al capital humano. Pese a ello, se echa en falta estudios de estas características en donde se haga especial referencia a la estimación de la rentabilidad de la educación de los jóvenes. 


\section{DisEÑO DE LA MUESTRA}

En este trabajo nos centramos en el estudio de los rasgos básicos de los jóvenes entre 16 y 34 años que se encuentran trabajando en el momento de la realización de la encuesta. Se hace especial énfasis en la relación existente entre la educación y los salarios con el fin de disponer de resultados que nos ayuden a entender mejor cuál es el verdadero valor añadido de la educación en el proceso de inserción laboral de la población de estas comarcas ${ }^{4}$.

La base de datos utilizada ha sido realizada entre los meses de marzo y abril de 2008 y metodológicamente cumple los siguientes requisitos:

1. Ámbito: Comarca de Bergantiños y zonas geográficas de Costa da Morte y Ferrolterra.

2. Universo: Población asalariada entre 16 y 34 años, ambos incluidos.

3. Tamaño muestral: 956 individuos. La estratificación de la muestra se realizó mediante una clasificación cruzada por ayuntamiento, grupos de edad y género.

4. El error muestral para cada una de las zonas fue del $\pm 2 \%$ para un nivel de confianza del $98 \%$.

5. Método: Polietápico con estratificación en la primera fase y afijación no proporcional entre las zonas y proporcional en los ayuntamientos.

Los totales muestrales tratan de que la muestra no baje de cuarenta y cinco unidades en cada ayuntamiento, de modo que se puedan hacer las estimaciones en cada municipio. También, se ha procurado que se mantenga autoponderada para los distintos grupos de edad, ya que se considera que es importante en el análisis las variaciones en el comportamiento y en la situación del empleo con distintos niveles de estudios.

Es un hecho conocido que el nivel educativo de los jóvenes españoles ha aumentado de forma muy rápida durante los últimos veinte años. Los datos disponibles sobre el

${ }^{4}$ La información a utilizar en este estudio forma parte de una investigación más genérica realizada por el Observatorio Ocupacional de la Universidad de A Coruña cuyo objetivo es analizar la situación educativa y laboral de los jóvenes gallegos de diversas zonas (Comarca de Bergantiños y zonas geográficas de Costa da Morte y Ferrolterra), y su transición desde el sistema educativo al mercado laboral. 
nivel de formación de la población española en edad de trabajar permiten también afirmar que existe un aumento significativo en los años de estudio de la población activa. En el cuadro 1 se presenta la distribución porcentual de los jóvenes encuestados por nivel de estudios y género. El nivel educativo lo clasificamos en los siguientes tramos:

- Nivel de estudios bajo: que se corresponde con Primaria, Graduado Escolar, ESO o sin estudios.

- Nivel de estudios medio: que se corresponde con BUP, FPI o ciclos de grado medio.

- Nivel de estudios anterior al superior: que se corresponde con COU, Bachillerato, FPII o ciclos de grado superior.

- Nivel de estudios superior: que se corresponde con el título de diplomado o licenciado.

CuADRo 1.-Distribución porcentual del nivel de estudios completado por grupos de edad y género

\begin{tabular}{|l|r|r|r|r|r|r|r|r|}
\hline & \multicolumn{4}{|c|}{ Hombre } & \multicolumn{4}{c|}{ Mujer } \\
\cline { 2 - 9 } & Bajo & Medio & $\begin{array}{c}\text { Ant. al } \\
\text { superior }\end{array}$ & Superior & Bajo & Medio & $\begin{array}{c}\text { Ant. al } \\
\text { superior }\end{array}$ & Superior \\
\hline $16-19$ & 90,2 & 9,8 & & & 92,9 & 7,1 & & \\
\hline $20-24$ & 50 & 35,4 & 11,0 & 3,6 & 30,2 & 34,8 & 16,7 & 18,3 \\
\hline $25-29$ & 25,5 & 14,6 & 33,6 & 26,3 & 16,2 & 18,8 & 27,4 & 37,6 \\
\hline $30-34$ & 31,2 & 16,0 & 23,6 & 29,2 & 21,5 & 14,8 & 30,7 & 33,0 \\
\hline Total & 40,3 & 18,6 & 21,5 & 19,6 & 27,3 & 20,3 & 23,8 & 28,6 \\
\hline
\end{tabular}

Con la información disponible se observa que las mujeres han completado estudios de nivel anterior al superior y superior en el 52,4\% de los casos, mientras que en los hombres este porcentaje es tan solo del $41,1 \%$. Es muy sorprendente el alto porcentaje de varones sin estudios o con un nivel de estudios bajo que alcanza el 40,3\%, mientras que en las mujeres esta proporción se reduce al $27,3 \%$.

Los datos sobre el nivel de estudios completados de los encuestados por género muestran diferencias significativas, sobre todo en el nivel de estudios bajo y superior. En el primer caso, para el nivel de estudios bajo, los varones superan a las mujeres en 13 puntos porcentuales, mientras que en los estudios superiores se presenta el caso opuesto, en el que las mujeres superan a los hombres en 9 puntos porcentuales (varones $19,6 \%$, mujeres $28,6 \%$ ). 


\section{RESULTADOS EMPÍRICOS}

Lo que ocurre en el mercado laboral y, en particular, con la demanda de trabajo de los empleadores, suele ejercer una influencia creciente en las personas cuando estas están entre los dieciséis y los treinta y cuatro años. A medida que pasa el tiempo, el joven intuye que sus decisiones educativas repercuten en su porvenir laboral, pero asimismo, el mercado de trabajo ejerce una influencia significativa sobre el sistema educativo que se estima de gran importancia. Esto no excluye el que existan otras variables también importantes, y además, no todos los individuos las valoran de la misma forma.

El mercado de trabajo para el joven es un dato. La demanda de trabajo de los empleadores se percibe por la juventud en términos de una gama de puestos de trabajo y de posibles carreras o trayectorias profesionales a las que los jóvenes creen poder acceder. En este panorama de posibilidades laborales, el individuo puede creer que el nivel educativo (obtenido o que espera alcanzar) es una condición necesaria o, quizás, una poderosa ayuda para lograr determinados puestos de trabajo.

Con el fin de dar información sobre la situación del mercado laboral de los jóvenes encuestados, en particular, de los rendimientos privados que los jóvenes obtienen de sus inversiones en educación y de la importancia que tienen otras variables potencialmente significativas, se utiliza el modelo de Mincer para corregir el problema de la endogeneidad de la educación a través de la técnica de variables instrumentales ${ }^{5}$. La variable dependiente utilizada es el logaritmo de los salarios y las independientes son aquellas que consideramos pudieran ser relevantes teniendo en cuenta el estudio descriptivo (ver apéndice).

Como punto de partida de este apartado se ha estimado por MCO, la clásica ecuación minceriana que relaciona el logaritmo del salario con la educación, la experiencia y su cuadrado. La finalidad de esta estimación es calcular cuál es el efecto marginal de cada año de educación en los salarios. Los resultados aparecen en el cuadro 2.

Los coeficientes estimados son significativos y presentan el signo adecuado, las aportaciones de la educación y de la experiencia a los ingresos son positivas, frente al coeficiente del cuadrado de la experiencia que es negativo.

${ }^{5}$ El problema de la selección muestral ya queda prácticamente reducido al tratarse de una muestra de trabajadores y tener conocimiento de sus ingresos laborales. 
Cuadro 2.-Estimación modelo de Mincer simple

\begin{tabular}{|l|c|c|c|}
\hline & Coeficientes & Error típico & T \\
\hline Constante & $5,934534^{* * *}$ & 0,0502 & 118,20 \\
\hline Escol & $0,053656^{* * *}$ & 0,0041 & 13,15 \\
\hline Exp & $0,042934^{* * *}$ & 0,0089 & 4,81 \\
\hline Exp2 & $-0,001188^{* *}$ & 0,0005 & $-2,34$ \\
\hline F & \multicolumn{3}{|c|}{} \\
Número de observaciones & \multicolumn{3}{|c|}{94,37} \\
$\mathrm{R}^{2}$ ajustado & 0,2623 & \\
\hline
\end{tabular}

Resultados corregidos de heterocedasticidad

** Coeficientes significativos al $5 \%$.

$* * *$ Coeficientes significativos al $1 \%$.

Los años de educación tienen un impacto positivo y significativo en los salarios, la tasa de rentabilidad de la educación es del 5,36\% lo que implica que los ingresos crecen a medida que aumenta el número de años de escolarización de los jóvenes encuestados.

En el modelo 2 (cuadro 3) se estima el modelo de Mincer ampliado en donde se analiza el efecto de otras características potencialmente explicativas como pueden ser, entre otras, el sexo, la edad, el estado civil o el sector económico en el que trabaja ${ }^{6}$. También se incluye otro tipo de variables, con el objeto de observar determinadas características, que normalmente no están incluidas en la mayoría de las investigaciones, como puede ser la falta de iniciativa del individuo hacia el autoempleo que figura en el modelo como «D_iniciat» y que recoge la preferencia del joven por ser autónomo si las condiciones salariales son las mismas, y un indicador de movilidad laboral, recogido en la variable «D_ubik2»y que se define como la preferencia por parte del individuo de ubicaciones del trabajo cercanas a su domicilio. Los resultados han sido los siguientes:

${ }^{6}$ Para ver la definición de las variables explicativas consultar Apéndice. 
CuAdro 3.-Estimación modelo de Mincer ampliado

\begin{tabular}{|l|c|c|c|}
\hline & Coeficientes & Error estándar & T \\
\hline Constante & 6,4593 & $0,2227^{* * *}$ & 29,01 \\
\hline Escol & $0,0449^{* * *}$ & 0,0046 & 9,68 \\
\hline Exp & $0,0267^{* * *}$ & 0,0098 & 2,72 \\
\hline Sexo & $0,1848^{* * *}$ & 0,0209 & 8,85 \\
\hline D_Ajena & $0,1092^{* * *}$ & 0,0411 & 2,66 \\
\hline D_ubik2 & $-0,1487^{* * *}$ & 0,0334 & $-4,45$ \\
\hline Edad_1 & $-0,4016^{*}$ & 0,2116 & $-1,90$ \\
\hline Adm_Públ & $0,0842^{* * *}$ & 0,0271 & 3,10 \\
\hline D_act_1 & $-0,1000^{* *}$ & 0,0503 & $-1,99$ \\
\hline D_iniciat & $-0,0731^{* *}$ & 0,0323 & $-2,26$ \\
\hline F & & 29,89 & \\
Número de observaciones & & 956 & \\
$\mathrm{R}^{2}$ ajustado & & 0,3872 & \\
\hline
\end{tabular}

Resultados corregidos de heterocedasticidad.

* Coeficientes significativos al $10 \%$.

** Coeficientes significativos al 5\%.

*** Coeficientes significativos al $1 \%$.

Respecto a los resultados de la estimación de la función de ganancias ampliada, es necesario señalar que la rentabilidad de los años de educación de los jóvenes encuestados disminuye a un 4,49\%. Este resultado indica que existen otras variables que influyen de manera significativa sobre los salarios además de la educación.

Asimismo, se observa que en este modelo la experiencia al cuadrado no es significativa. La explicación más plausible de este hecho es que la población objeto de estudio son los jóvenes entre 16 y 34 años, y en la mayoría de los casos su experiencia es pequeña o muy pequeña, con una reciente incorporación al mercado laboral lo que provoca que no existan entre ellos diferencias significativas en términos de experiencia laboral. Es razonable pensar que una vez descontado el efecto de la educación, las diferencias en sus salarios se deban a otro tipo de factores.

Respecto al resto de variables estudiadas, se observa que ser hombre frente a ser mujer tiene un efecto positivo y significativo en los salarios, lo mismo que la experiencia, el trabajar por cuenta ajena, y el trabajar para la Administración Pública.

De las variables que hemos considerado y que son significativas, las que afectan negativamente a los salarios son: el pertenecer al grupo de edad menor de 19 años, 
el elegir trabajos que se encuentren en la misma ubicación o estén cercanos al lugar de residencia, pertenecer al sector de la agricultura, comercio, hostelería, transporte e industria y el preferir ser autónomo.

Sorprende el hecho de que la variable «preferencia por ser autónomo» (D_iniciat) afecte negativa y significativamente a los ingresos de los individuos. Una de las explicaciones de este hecho puede ser que, en muchos casos el planteamiento de ser autónomo viene promovido por una situación de inestabilidad laboral, condiciones precarias en el trabajo y sueldos bajos o muy bajos.

Se analiza a continuación la posible endogeneidad de la educación. Como ya mencionamos en el apartado 2, uno de los métodos más utilizados para solventar el problema de la correlación entre los términos de perturbación de las ecuaciones de ingresos y de la escolaridad, consiste en identificar un conjunto de variables instrumentales que afecten a la escolaridad pero no a los ingresos.

Para resolver este sesgo nos hemos apoyado en los trabajos de Blackburn y Neumark (1995), Brunello y Miniaci (1999) y Uusitalo (1999), en todos ellos se utilizan como variables instrumentales el background familiar (nivel educativo de los padres, tipo de trabajo de los mismos, etc).

En la investigación se han tomado como variables instrumentales el nivel educativo de los padres y el vivir en un municipio de más de 6.000 habitantes $^{7}$. Al aplicar el «test de Hausman» se confirma que la variable educación es endógena, lo que implica que el método más apropiado para calcular los rendimientos de la educación es por «mínimos cuadrados en dos etapas» $(\mathrm{MC} 2 \mathrm{E})^{8}$ en donde la variable educación es sustituida por las variables instrumentales mencionadas anteriormente (cuadro 4).

Antes de interpretar los resultados es importante tener en cuenta que, si bien es cierto que se rechaza la hipótesis nula de exogeneidad, dicho rechazo es por un pequeño margen, lo que unido al hecho del déficit de variables instrumentales nos hace tener precaución con dichas interpretaciones.

La conclusión más significativa que se puede obtener con esta estimación es el aumento en la rentabilidad de la educación que pasa de un 5,36\% en el modelo de Mincer simple a un 7,49\% en la estimación con variables instrumentales. Estos datos concuerdan con la mayoría de los estudios previos en donde la elevación en los rendimientos

${ }^{7}$ La fuente estadística utilizada adolece de variables que nos permitan definir de una forma más completa el background familiar de los jóvenes encuestados.

${ }^{8}$ En adelante Mínimos Cuadrados en dos etapas se reflejará como MC2E. 
de la educación al incluir variables instrumentales es un hecho constatado (Angrist y Krueger, 1991; Card, 1994; Barceinas, y cols., 2001 y Lillo y Casado, 2010).

CUADro 4.-Estimación mínimo cuadrado en dos etapas (MC2E)

\begin{tabular}{|c|c|c|c|}
\hline & Coeficientes & Error típico & $\mathrm{z}$ \\
\hline Constante & $6,0070^{* * *}$ & 0,114 & 52,65 \\
\hline Escol & $0,0749^{* * *}$ & 0,014 & 5,26 \\
\hline Experiencia & $0,0288^{* * *}$ & 0,011 & 2,63 \\
\hline Sexo & $0,2107^{* * *}$ & 0,021 & 10,11 \\
\hline Edad_1 & $-0,4143^{* * *}$ & 0,039 & $-10,64$ \\
\hline Edad_2 & $-0,3223^{* * *}$ & 0,063 & $-5,11$ \\
\hline Edad_3 & $-0,3421^{* * *}$ & 0,969 & $-3,53$ \\
\hline Edad_4 & $-0,3929^{* * *}$ & 0,1182 & $-3,32$ \\
\hline $\begin{array}{l}\text { F } \\
\text { Número de observaciones } \\
\mathrm{R}^{2} \text { ajustado } \\
\text { Test de Hausman: }\end{array}$ & & $\begin{array}{c}1 . e+307 \\
956 \\
0,3177 \\
61 \text { p-valor: } 0 \text {, }\end{array}$ & \\
\hline
\end{tabular}

*** Coeficientes significativos al $1 \%$.

En esta investigación no se ha tenido en cuenta el problema de la selección muestral ya que al tratarse de una muestra cuya población objeto de estudio son los jóvenes entre 16 y 34 años que trabajan, el sesgo derivado de la imposibilidad de conocer los ingresos de aquellos individuos que no están trabajando y de los que, por lo tanto, no disponemos de datos es inexistente.

Las estimaciones de la ecuación de Mincer obtenidas implican que existe una única tasa de rendimiento de la educación, pero tanto la teoría como la evidencia empírica sugieren que si se desagrega la escolaridad de una forma más flexible se obtienen unos rendimientos del proceso de inversión en educación más adecuados (Sapelli, 2009). Es por ello, que en el siguiente modelo se desagrega la educación por nivel de estudios distinguiendo entre nivel bajo, medio, anterior al superior y superior ${ }^{9}$ (cuadro 5).

${ }^{9}$ Esta estructura conocida como «spline» nos permite obtener una mejor caracterización de las tasas de retorno a la educación. 
¿SIGUE SIENDO RENTABLE LA EDUCACIÓN?

Cuadro 5.-Estimación modelo de Mincer ampliado. Desagregación por niveles educativos

\begin{tabular}{|l|c|c|c|}
\hline & Coeficientes & Error estándar & T \\
\hline Constante & 6,8875 & $0,2179^{* * *}$ & 31,60 \\
\hline Est_3 & 0,1458 & $0,0291^{* * *}$ & 5,02 \\
\hline Est_4 & 0,2825 & $0,034^{* * *}$ & 8,30 \\
\hline Exp & $0,0260^{* * *}$ & 0,0100 & 2,58 \\
\hline Sexo & $0,1914^{* * *}$ & 0,0212 & 9,04 \\
\hline D_Ajena & $0,1072^{* * *}$ & 0,0411 & 2,61 \\
\hline D_ubik2 & $-0,1525^{* * *}$ & 0,0338 & $-4,51$ \\
\hline Edad_1 & $-0,4013^{*}$ & 0,2078 & $-1,93$ \\
\hline Adm_Públ & $0,0836^{* * *}$ & 0,0274 & 3,05 \\
\hline D_act_1 & $-0,1186^{* *}$ & 0,0506 & $-2,34$ \\
\hline D_iniciat & $-0,0747^{* *}$ & 0,0321 & $-2,33$ \\
\hline F & & 26,53 & \\
Número de observaciones & & 956 & \\
R $^{2}$ ajustado & & 0,3874 & \\
\hline
\end{tabular}

Resultados corregidos de heterocedasticidad.

* Coeficientes significativos al $10 \%$.

** Coeficientes significativos al $5 \%$.

$* * *$ Coeficientes significativos al $1 \%$.

En el cuadro 5 se presentan los resultados obtenidos para el modelo de Mincer ampliado, teniendo en cuenta la desagregación de la escolaridad de acuerdo al nivel de educación completado. Se observa que los coeficientes mantienen el signo y la significación correctas. El poseer niveles de estudios anterior al superior y superior afectan de manera positiva y significativa a los salarios. En particular, destaca el alto rendimiento de la educación de los estudios superiores, alrededor de un $28,2 \%{ }^{10}$, casi el doble de la rentabilidad que obtienen los entrevistados que poseen estudios de nivel anterior al superior (14,6\%). El resto de variables significativas, tal y como era de esperar, tienen unos coeficientes muy similares al modelo de Mincer ampliado.

${ }^{10}$ Es en este nivel educativo en donde la falta de incorporación de los costes directos asociados a dichos estudios pueden tener un mayor impacto en el resultado de dicho rendimiento (Sapelli, 2003). 


\section{CONCLUSIONES}

En este trabajo se pretende ampliar el conocimiento ya existente sobre la evaluación de los rendimientos de la educación, aplicado en este caso particular a los jóvenes. Para el cálculo de los rendimientos se utilizaron las ecuaciones de ingresos de Mincer. A partir de una muestra de jóvenes entre 16 y 34 años de Galicia (de Ferrolterra, Bergantiños y Costa da Morte), se evidencia la relación existente entre la escolaridad y los ingresos laborales de los jóvenes.

Previamente a hablar de las conclusiones sobre los rendimientos de la educación, para el grupo objeto de estudio, decir que en lo referente al nivel educativo alcanzado, las mujeres han completado estudios de nivel anterior al superior y superior en el $52,4 \%$, mientras que en los hombres este porcentaje alcanza sólo el $41,1 \%$. Es sorprendente el alto porcentaje de varones sin estudios o con un nivel de estudios bajo que alcanza el 40,3\%, mientras que en las mujeres esta proporción se reduce al 27,3\%.

La relación entre rendimientos y escolarización (medida como años de estudio completados) muestra un rendimiento de la educación del 5,36\%, un poco más bajo que los resultados de las investigaciones revisadas, pero es necesario recordar que al tratarse de jóvenes su situación laboral es más deficiente que las del resto de la población, lo que afecta no sólo a sus dificultades de inserción laboral, sino a sus ingresos.

Cuando analizamos el efecto de otras variables potencialmente explicativas, el rendimiento de la educación baja hasta un 4,49\%, lo que indica que existen otras variables que afectan de manera significativa a los salarios. Las variables que afectan positivamente a los salarios son el sexo, el trabajar por cuenta ajena y el trabajar para la Administración Pública. Por otro lado, las que influyen negativamente en los salarios son pertenecer al grupo de edad de menos de 19 años, la preferencia por ser autónomo, trabajar en la misma localidad de residencia, y desarrollar la actividad en el sector de la agricultura, comercio, hostelería, transporte, comunicación e industria.

El test de Hausman confirma por un pequeño margen que la variable educación es endógena, por ello, se utiliza el método de estimación mínimo cuadrado en dos etapas (MC2E), en donde las variables instrumentales utilizadas son el nivel educativo de los padres y el vivir en un municipio con más de 6.000 habitantes. Los resultados confirman los aumentos en rentabilidad de la educación que alcanza un 7,49\%.

Asimismo, se ha desagregado la escolaridad por niveles (nivel de estudios bajo, medio, anterior al superior y superior) para calcular de una forma más flexible los rendimientos del proceso de inversión en educación. Los resultados muestran un ren- 
dimiento de la educación de poseer estudios superiores del $28,25 \%$ y del 14,6\% para aquellos que poseen estudios anteriores al superior.

Una vez analizados diferentes modelos para la estimación de la función de ganancias, se constata que son bastantes representativas del salario, ya que explican alrededor de un 39\% de las variaciones salariales observadas para los jóvenes en los modelos de Mincer ampliados y un 32\% para el caso de MC2E. Este porcentaje es relativamente elevado, pues no hay que olvidar que la estructura de los salarios depende de un número importante de factores que no están explícitamente reflejados en el modelo estimado y más en particular, en nuestro objeto de investigación, con toda la problemática asociada a la inserción laboral de los jóvenes.

\title{
APÉNDICE.-DEFINICIÓN DE VARIABLES
}

\author{
Escol \\ Años de educación escolar completados. \\ Exp \\ Experiencia laboral del individuo medida en años. \\ Exp2 \\ Experiencia al cuadrado. \\ Sexo \\ Variable dicotómica cuyo valor de referencia es ser hombre. \\ Edad_1 \\ Variable dicotómica cuyo valor de referencia es que la edad del \\ individuo sea menor o igual a 19 años. \\ Edad_2 \\ Variable dicotómica cuyo valor de referencia es que la edad del \\ individuo esté entre 20 y 24 años. \\ Edad_3 \\ Variable dicotómica cuyo valor de referencia es que la edad del \\ individuo esté entre 25 y 29 años. \\ Edad_4 \\ Variable dicotómica cuyo valor de referencia es que la edad del \\ individuo esté entre 30 y 34 años. \\ Civil_1 \\ Variable dicotómica cuyo valor de referencia es tener pareja. \\ D_Ajena \\ Variable dicotómica cuyo valor de referencia es trabajar por cuenta \\ ajena. \\ Adm_Públ Variable dicotómica cuyo valor de referencia es trabajar para la \\ administración pública. \\ D_act_1 \\ Variable dicotómica cuyo valor de referencia es trabajar para los sectores \\ de la agricultura, comercio, hostelería, transporte, comunicación e \\ industria. \\ D_act_2 \\ Variable dicotómica cuyo valor de referencia es trabajar para el sector de \\ construcción, actividades inmobiliarias y de alquiler \\ D_act_3 \\ Variable dicotómica cuyo valor de referencia es trabajar en el sector de \\ finanzas (bancos, aseguradoras, etc).
}


D_act_4 Variable dicotómica cuyo valor de referencia es trabajar en el sector de educación.

D_act_5 Variable dicotómica cuyo valor de referencia es trabajar en el sector de sanidad.

D_iniciat Variable dicotómica cuyo valor de referencia es pensar alguna vez en montar un negocio propio.

D_ubik2

Variable dicotómica cuyo valor de referencia es preferir ser autónomo con las mismas condiciones salariales.

Las variables instrumentales incluidas en el modelo para comprobar la endogeneidad de la educación han sido:

Padre con estudios Variable dicotómica cuyo valor de referencia es que los padres posean como mínimo estudios de nivel medio.

D_conc_2 Variable dicotómica cuyo valor de referencia es trabajar en un concello de más de 6.000 habitantes.

\section{REFERENCIAS BIBLIOGRÁFICAS}

Alba Ramírez, A. y San Segundo, M. J. (1995), «The returns to education in Spain», Economics of Education Review, vol 14, págs. 155-166.

Angrist, J. y Krueguer, A. (1994), «Split-sample instrumental variables», Technical Paper, 150, Cambridge, Mass, NBER.

- (1995), «Split-Sample instrumental variables Estimates of the Return to Schooling», Journal of Business and Economics Statistics, 13, págs. 225-235.

Arrazola, M. y Hevia, J. de (2001), «Rendimiento de la educación en España: nueva evidencia de las diferencias entre hombres y mujeres», Papeles de Trabajo del Instituto de Estudios Fiscales. Serie economía, núm. 24, págs. 1-28.

Arrazola, M.; Hevia, J. de; Risueño, M. y Sanz, J. F. (2003), «Returns to education in Spain: some evidence of the endogeneity of schooling», Education Economics, vol. 11 (3), págs. 293-304.

Ashenfelter, O. y Rouse, C. (1997), Income, schooling, and ability: Evidence from a new sample of identical twins, Working Paper 6106, Cambridge, Mass, NBER.

Asplund, R. y Pereira, P. (1999), Returns to human capital in Europe. A literature review, Helsinki, ETLA.

Barceinas, F.; Oliver, J.; Raymond, J. L. y RoIG, J. L. (2000), «Los rendimientos de la educación y la inserción laboral en España», Papeles de Economía Española, núm. 86, págs. 128-148. 
Barceinas, F.; Oliver, J.; Raymond, J. L. y Roig, J. L. (2001), «Spain», en Harmon, C., Walker, I. y Westergaard-Nielsen, N. (eds.); Education and Earnings in Europe. A Cross Country Analysis of the Returns to Education, Edward Elgar, Cheltenham, págs. 234-264.

BECKER, G. S. (1964), Human capital: A theoretical and empirical analysis, with special reference to education, National Bureau of Economic Research, Nueva York.

Blackburn, M. y Neumark, D. (1993), «Omitted-Ability bias an the increase in the return to schooling», Journal of Labor Economics, 11, págs. 521-544.

- (1995), «Are ols estimates of the return to schooling biased down-ward? Another look», Review of Economics and Statistics, 77, págs. 217-230.

Brunello, G. y Miniaci, R. (1999), «The economic return to schooling for Italian men. An evaluation based on instrumental variables», Labour Economics, 6, págs. 509-519.

Calvo, J. L. (1988), «Rendimientos del capital humano en educación en España», Investigaciones Económicas, 12, págs. 473-482.

CANTILlon, R. (1775), Essay sur la nature du comerse en general, Hardcover.

CARD, D. (1994), Earnings, schooling, and ability revisited, Working Paper 4832, Cambridge, Mass., NBER.

- (2001), «Estimating the return to schooling: progress on some persistent econometric problems», Econometrica, 69, págs. 1127-1160.

Caparrós, A.; Gamero, C.; Marcenario, O. D. y Navarro, M. L. (2001), «Un análisis comparativo del rendimiento de la educación en España», X Jornadas de la Asociación de Economía de la Educación, Murcia.

Chon, E. y Addison, J. (1998), «The economics returns to lifelong learning in oecd countries», Education Economics, 6, págs. 253-307.

De Pablos, L. y Gil, M. (2005), «Los rendimientos y la productividad de la educación», Presupuesto y Gasto Público, núm. 39, págs. 49-72.

EAton, J. y Rosen, H. (1980), «Taxation, human capital and uncertainty», American Economic Review, 70, págs. 705-715.

Freire, J y Teijeiro, M. (2005), «La inserción de los jóvenes en Galicia. Una estimación minceriana de la rentabilidad de la educación», Cuadernosd e Economía, vol. 28, págs. 57-80.

- (2009), «Análisis que afectan a la decisión de ser emprendedor», Cuadernosd e Economía, vol. 32, págs. 5-28.

Griliches, Z. (1977), «Estimating the returns to schooling: some econometric problems», Econometrica, 45 , págs. 1-22.

Harmon, C.; Walker, I. y WestergaARd-Nielsen, N. (2001), Education and earnings in Europe. A Cross Country Analysis of the Return to Education, Cheltenham, Edward Elgar.

Harmon, C.; Oosterbeek, H. y Walter, I. (2003), «The returns to education: microeconomics», Journal of Economic Surveys, 17, págs. 115-155. 
Heckman, J. (1979), «Sample selection bias as a specification error», Econometrica 47(1).

HeCKMAN, J. y VYTLACIL, E. (2000), Identifying the role of cognitive ability in explaining the level of and change in the return to schooling, Working Paper 7820, Cambridge, Mass., NBER.

KodDE, D. y Ritzen, J. (1984), «Integrating investment and consumption motives in a neoclassical model of demand for education», Kyclos, 37, págs. 598-608.

Lassibille, G. y Navarro, M. L. (1998), «The evolution of returns to education in spain 19801991», Education Economics, vol. 6, págs. 3-9.

LEVhaRI, D. y WeIss, Y. (1974), «The effect of risk on the investment in human capital», American Economic Review, 64, págs. 950-973.

Lillo, A. y CASADO, J. (2010), «Endogeneidad de la educación e instrumentos para la estimación del rendimiento educativo en España», Investigaciones Económicas, págs. 451-458.

Marcenaro, O. D. y Navarro, M. L. (2005), «Nueva evidencia sobre el rendimiento del capital humano en España», Revista de Economía Aplicada, núm. 37, págs. 69-88.

Mincer, J. (1974), Schooling, experience and earnings, National Bureau of Economic Research, New York.

Murnane, R.; Willett, J. y LeVy, F. (1995), «The growing importance of cognitive skills in wage determination», Review of Economics and Statistics, 77, págs. 251-266.

OCDE (1998), Human capital investment. an international comparison, OCDE, París.

Oliver, J.; Raymond, J. L.; Roig, J. L. y Roca, A. (1998), «Función de ingresos y rendimiento de la educación en España 1990», Documentos de Trabajo de la Fundación de las Cajas de Ahorros Confederadas, núm. 138/1998, Fundación FUNCAS, Madrid.

PARSONS, D. O. (1972), «Specific human capital: An application to quit rates and layoff rates», Journal of Political Economy, vol. 80, págs. 1120-1143.

Pons, E. y GonZalo, M.T. (2002), «Returns to schooling in Spain: How reliable are instrumental variable estimates?», Labour, vol. 16, págs. 747-770.

Pscharopoulos, G. (1994), «Returns to investment in education: A global update», World Development, vol. 22, págs. 1325-1343.

Salas, M. (2004), «Rendimientos privados de las inversiones en educación superior a partir de las ecuaciones de ingresos», Hacienda Pública Española/Revista de Economía Pública, núm. 169, págs. 87-117.

- (2008), Economía de la educación, Pearson Prentice Hall, Madrid.

San Segundo, M. J. (1996), «Educación e ingresos en el mercado de trabajo español», Cuadernos Económicos de ICE, núm. 63, págs. 105-125.

SAPELLI, C. (2003), Ecuaciones de Mincer y las tasas de retorno a la educación en Chile: 1990-1998, Documento de Trabajo 254, Instituto de Economía, Pontificia Universidad Católica de Chile.

- (2009), Los retornos a la educación en Chile: Estimaciones por corte transversal y por cohortes, Documento de trabajo 349, Instituto de Economía, Pontificia Universidad Católica de Chile. 
Schultz, T. W. (1961), «Education and economic growth», en Henry, N.B. (ed.): Social Forces Influencing American Education, University of Chicago Press, Chicago, págs. 46-88.

Sмiтн, A. (1776), La riqueza de las naciones, Orbis.

Uusitalo, R. (1999), «Return to education in Finland», Labour Economics, 6, págs. 569-580.

VILA, L. y MorA, J. G. (1996), «Educación e ingresos de los trabajadores en España: Evolución en los años ochenta», en J. Grao y A. Ipiña (eds.), Economía de la educación. Temas de estudio e investigación, Servicio Central de Publicaciones del Gobierno Vasco, Colección Estudios y Documentos, núm. 22, págs. 233-257.

- (1998), «Changing Returns to education in Spain during the 1980s», Economics of Education Review, vol. 17, págs. 173-178.

Wallace, T. e IHnen, L. (1975), «Full-Time schooling in life-cycle models of capital accumulation», Journal of Political Economy, 83, págs. 137-155. 